\title{
Hypothetical protein Cpn0423 triggers NOD2 activation and contributes to Chlamydia pneumoniae-mediated inflammation
}

Hong-liang Chen ${ }^{1,2}$, Guo-zhi Dai ${ }^{1,2}$, An-wen Zhou ${ }^{1}$, Ran-hui Li ${ }^{3}$, Hong-xia Yuan ${ }^{1}$, Jing Xiang ${ }^{1,2}$, Xiao-xing You ${ }^{3}$, Ou $\operatorname{Ran}^{3}$ and Yi-mou Wu W $^{3 *}$

\begin{abstract}
Background: Chlamydia pneumoniae (C. pneumoniae) is pathogenic to humans, by causing pulmonary inflammation or bronchitis in both adolescents and young adults. However, the molecular signals linking C. pneumoniae components to inflammation remain elusive. This study was to investigate the effect of Chlamydia-specific Cpn0423 of $C$. pneumoniae on C. pneumoniae-mediated inflammation.

Results: Cpn0423 was detected outside of C. pneumoniae inclusions, which induced production of several cytokines including macrophage inflammatory protein-2 (MIP-2) and interleukins (ILs). Production of the Cpn0423-induced cytokines was markedly reduced in cells pretreated with NOD2-siRNA, but not with negative control oligonucleotides. Mice treated with Cpn0423 through intranasal administration exhibited pulmonary inflammation as evidenced by infiltration of inflammatory cells, increased inflammatory scores in the lung histology, recruitment of neutrophils and increased cytokines levels in the BALF.
\end{abstract}

Conclusion: Cpn0423 could be sensed by NOD2, which was identified as an essential element in a pathway contributing to the development of C. pneumoniae -mediated inflammation.

Keywords: Chlamydia pneumoniae, Cpn0423, NOD2, Inflammatory

\section{Background}

Chlamydia pneumoniae (Cpn), a common non-viral intracellular pathogen, is known as a leading cause of human respiratory tract infections worldwide, and responsible for up to $10 \%$ of community acquired pneumonias [1]. C. pneumoniae mainly infects the mucosal surfaces of human respiratory tract, causing various respiratory diseases, such as pneumonia, bronchitis and pharyngitis $[2,3]$. It is also associated with the development of or exacerbation of chronic obstructive pulmonary disorder [4]. C. pneumoniae undergoes a unique biphasic developmental form, including the noninfectious metabolically active reticulate body $(\mathrm{RB})$ and

\footnotetext{
* Correspondence: yimouwu@sina.com

${ }^{3}$ Institute of Pathogenic Biology, Medical College, University of South China, Hengyang 421001, China

Full list of author information is available at the end of the article
}

the infectious elementary body (EB) [5]. Upon internalization of C. pneumoniae by a host cell, it is believed to trigger immune responses and induce chronic inflammation with the release of cytokines such as IL-6, IL-10 and TNF-alpha $[6,7]$. Due to this link with important chronic inflammatory diseases, and the fact that $C$. pneumoniae infection occurs worldwide with a seroprevalence of $70 \%$ in the adult population $[8,9]$, understanding the mechanisms underlying inflammatory response during C. pneumoniae infection is of great importance to dissect its potential role in these chronic inflammatory diseases.

C. pneumoniae differs from most bacteria in that it lacks the ability to produce its own adenosine triphosphate (ATP). Thus, C. pneumoniae has evolved the ability to secrete proteins into both the inclusion membrane and host cell cytoplasm, which may help the organisms 
of C. pneumoniae to take up nutrients and energy from host cells, and to maintain the integrity of host cells by preventing the infected host cells from host immune response [10, 11]. Cpn0423 is a Chlamydia-specific hypothetical protein which is highly conserved among all chlamydial genomes [12], suggesting an important role of Cpn0423 in maintaining C. pneumoniae intracellular infection.

Innate immune system represents the first line of defense against foreign pathogens, such as bacteria, viruses and fungi $[13,14]$. The two principal families of pathogen detectors are the Toll-like receptors (TLRs) and the nucleotide-binding oligomerization domain-like receptors (NLRs) [15]. TLRs are embedded in the plasma membrane and recognize conserved pathogenassociated molecules. Studies from our laboratory indicated that heat shock protein 10 (HSP10) of C. pneumoniae could elicit inflammatory reactions mediated by TLR4 [16]. Others also confirmed that both TLR2 and TLR4 signaling induce early cytokine and chemokine production during C. pneumoniae respiratory infection [17]. Surprisingly, the IFN- $\gamma$ could be induced by $C$. pneumoniae in the absence of TLR4/MyD88 signaling pathways [18], suggesting a potential contribution of intracellular recognition receptors like NLRs to C. pneumoniae-induced inflammatory cytokines production. NLRs, also called caspase recruitment domaincontaining proteins, are a group of evolutionarily conserved intracellular pattern recognition receptors (PRRs) that play a vital role in innate immune responses against certain bacterial infections $[15,19]$. Nucleotidebinding oligomerization domain-containing protein 2 (NOD2) is a large multi-domain protein, that serves as a general sensor for both intracellular Gram-positive and Gram-negative bacteria and that mediates responsiveness to molecules in the cytoplasm originated from bacteria, including muramyl dipeptide conserved in peptidoglycans [20]. NOD2 generally prevails in the cytoplasm in a dormant form. Once activated, it will elicit specific immune responses by the recruitment of specific adaptor molecules as well as effector molecules like inflammatory procaspases and kinases [21]. Although NOD2 was reported to be involved in the activation of innate immune defenses against intracellular C. pneumoniae [6], further details of the molecular signals linking NOD2 to $C$. pneumoniae infection remains largely unknown.

Our previous studies already identified that Cpn0423 protein was expressed and located in C. pneumoniae infected host cell cytosol. Since NOD2 was also one of the cytosolic PRRs, we hypothesized that Cpn0423 is a potent activator that stimulates specific inflammatory responses via a NOD2-dependent mechanism. We treated bone marrow-derived macrophages (BMDMs) with Cpn0423 and measured cytokine responses. We assessed the specific roles of NOD2 signaling by using NOD2-siRNA oligonucleotides. We demonstrated the pro-inflammatory properties of $\mathrm{Cpn} 0423$ in a mouse model in vivo. Finally, we found that Cpn0423 acts as a signal for NOD2 receptor, which provides important information on the mechanism of $C$. pneumoniae pathogenesis.

\section{Methods}

\section{Organism and C. pneumoniae infection}

C. pneumoniae strain AR39 was kindly provided by Dr. Zhong (University of Texas, San Antonio, TX). C. pneumoniae was propagated, purified and quantified as previously described [22]. For infection, HEp-2 cells were determined to be free of Mycoplasma contamination, and inoculated with C. pneumoniae AR39 organisms at an MOI of 0.5 in the presence of $2 \mu \mathrm{g} / \mathrm{ml}$ of cycloheximide, and grown in RPMI 1640 medium supplemented with $10 \%$ FBS at $37{ }^{\circ} \mathrm{C}$ in a $5 \% \mathrm{CO} 2$ environment for $72 \mathrm{~h}$ as indicated in the following experiments.

\section{C. pneumoniae gene cloning and fusion protein expression} Gene coding for the ORF Cpn0423 was amplified from $C$. pneumoniae AR-39 genomic DNA and cloned into pGEX vectors (TaKaRa, Dalian, China). The special primers for full-length Cpn0423 covering residues (condon 1-429) were as follows: forward primer 5'-CGC-GGATCC (BamH I) -ATGTTGGATAATGAATGGAA AGC-3', backward primer $5^{\prime}$-TTTTCCTTTT-GCGGCCGC (Not I) -TTAACGAACTAA CGCAGCATTT-3'; The gene Cpn0423 was expressed as a fusion proteins with GST fused to the $\mathrm{N}$-terminus of the chlamydial proteins as previously described [23]. Overexpression of the fusion protein was induced by $0.2 \mathrm{mM}$ Isopropyl $\beta$-DThiogalactoside (IPTG) (TaKaRa). The recombinant fusion protein was extracted by lysing the E. coli BL21 (DE3) (Novagen) transformed with pGEX-6P-Cpn0423 recombinant plasmid via sonication in a Triton-X100 lysis buffer (1\% Triton X-100, $1 \mathrm{mM}$ phenylmethanesulfonyl fluoride, $75 \mathrm{U} / \mathrm{ml}$ aprotinin). After a high-speed centrifugation to remove debris, the fusion protein was further purified by affinity chromatography using Glutathione Sepharose 4B from ameshame pharmacia (Zkcy, Beijing, China) and was analyzed by SDS-polyacrylamide gel electrophoresis (SDS-PAGE) and Coomassie Blue staining. Pierce High-Capacity Endotoxin Removal Resin (BXGK, Beijing, China) was used to remove endotoxin from purified Cpn0423 fusion protein following its protocol. Final endotoxin concentration of purified Cpn0423 fusion protein used in this study was less than $60 \mathrm{pg} / \mathrm{mL}$, determined by Limulus amebocyte lysate kit (BXGK) based on a standard curve. The endotoxin-removed Cpn0423 fusion protein was used to immunize mice for producing 
polyclonal antibody (pAb) as previously described [24]. Briefly, mice were immunized three times intramuscularly with $100 \mu \mathrm{g}$ fusion protein and incomplete Freund's adjuvant (IFA) and three times intravenously with $100 \mu \mathrm{g}$ fusion protein without IFA.

\section{Immunofluorescence assay}

The immunofluorescence assay was carried out as described previously $[22,25]$. Briefly, Hep-2 cells, with C. pneumoniae infection grown on coverslips were fixed with $2 \%$ paraformaldehyde (Sigma) for $30 \mathrm{~min}$ at room temperature (RT), followed by permeabilization with $1 \%$ saponin (Sigma) for an additional $30 \mathrm{~min}$. After washing and blocking, the cell samples were subjected to antibody and chemical staining. Hoechst (Sangon Biotech, Shanghai, China) was used to visualize DNA. A rabbit anti-C. pneumoniae organism antibody (raised with $C$. pneumoniae AR39 organisms, unpublished data) and a goat anti-rabbit IgG secondary antibody conjugated with Cy2 (green; Sangon Biotech) were used to visualize $C$. pneumoniae AR39 organisms. A mouse anti-Cpn0423 antibody (raised with the Cpn0423 fusion protein, unpublished data) and a goat anti-mouse IgG conjugated with Cy3 (red; Sangon Biotech) were used to visualize Cpn0423 antigen. Uninfected Hep-2 cells were stained for control. In addition, for antibody absorption experiments, the primary antibodies were further absorbed with either the corresponding or heterologous fusion proteins CPAF [26] immobilized onto glutathioneconjugated agarose beads prior to staining, which was used to prove the antibody binding specificities. The absorption was carried out by incubating the antibodies with bead-immobilized antigens for $1 \mathrm{~h}$ at room temperature followed by pelleting the beads. The remaining supernatants were used for immunostaining. The immunofluorescence images were acquired using an ECLIPSE TE2000-5 fluorescence microscope (Nikon, Inc., Japan) connected to a Sony $3 \mathrm{CCD}$ color video camera.

\section{Cell isolation and culture}

Mouse bone marrow derived macrophages (BMDMs) were prepared as described previously with minor modifications [27]. Briefly, bone marrow cells were harvested from the tibias and femurs of mice, re-suspended in RPMI 1640 medium supplemented with $10 \mathrm{ng} / \mathrm{ml}$ macrophage colony-stimulating factor (TaKaRa), 10\% FBS (Invitrogen), 5\% horse serum (Invitrogen) and 1\% penicillin/streptomycin (Sigma), seeded in a 96-well plate $\left(2 \times 10^{5}\right.$ cells/well $)$ or 6 -well plate $\left(2 \times 10^{6}\right.$ cells/ well) and incubated in $\mathrm{CO}_{2}$ incubator at $37{ }^{\circ} \mathrm{C}$. Three days later, culture media were changed and the macrophages were ready for experiments at day 5 .

\section{Treatments of cell cultures}

Cultured BMDMs were washed twice, and then transferred to a pre-warmed serum-free RPMI 1640 medium containing $0.05 \%$ BSA for $1 \mathrm{~h}$ before treatment. Cpn0423 was complexed with Pro-Ject ${ }^{\text {tw }}$ Protein Transfection Reagent (PTR) (Invitrogen) following the manufacture's instruction and was incubated for $5 \mathrm{~min}$ at room temperature before applied to cell cultures. The cell cultures were then treated in RPMI 1640 supplemented with 10\% FBS. For additional control experiments, Cpn0423 or LPS was used in combination with $50 \mu \mathrm{g} / \mathrm{ml}$ Polymyxin B (PMB), as a LPS neutralizer [28] for $10 \mathrm{~min}$ before mixed with Pro-Ject ${ }^{\text {tu }}$ Reagent. For RNA interference experiments, small interfering RNA against NOD2 (NOD2-siRNA) and small interfering RNA negative control for NOD2 (NOD2-Con-siRNA) were transfected into BMDMs with Lipofectamine 3000 (Invitrogen), incubated at $37{ }^{\circ} \mathrm{C}$ for $48 \mathrm{~h}$ before applied to cell cultures. NOD2siRNA and NOD2-Con-siRNA oligonucleotides sequence were as follows: Sense 5'-CGGUGAAAGCGAAUG GAUU-3', Antisense 5'-AAUCCAUUCGCUUUCAC CG-3', Control (1) 5' -CGTGAGAGGGGGGACACUU3', Control (2) 5' -AAATCAC TTACTGGCGCTCG-3'.

\section{Cytokine protein measurement}

Culture medium and bronchoalveolar lavage fluid (BALF) were prepared and stored at $-80{ }^{\circ} \mathrm{C}$. ELISA kits ( $R \& D$ systems, Minneapolis, $M N$ ) were used to measure the levels of MIP-2, IL- 6 and TNF- $\alpha$, while the final cytokine concentrations were calculated based on a standard curve constructed in each experiment.

\section{Quantitative RT-PCR}

Quantitative RT-PCR was performed as described previously [27]. After total RNA isolation and cDNA Synthesis, real-time RT-PCR was performed by using the Power SYBR Green Master Mix (Applied Biosystems, CA) according to the manufacturer's protocols. For RTPCR experiments, $1 \mu \mathrm{g}$ of total RNA was reversetranscribed using SuperScript III reverse transcriptase (Invitrogen) prior to PCR amplification. The sequences of the oligonucleotide primers utilized for real time RTPCR were as follows: for NOD2, forward primer $5^{\prime}$ CAAACCCTCAGGATAGGAAATGTAG-3', backward primer 5' - TAAGTGAAGAGTCAGGTGATGGATG-3'; for TLR2, forward primer 5'-GGTTTCTGAGCCAG TACGAGTGTG-3', backward primer 5' - GGTAGTTC CCTGACGTGCTGTAGA-3'; for GAPDH, forward primer 5'-AACTTTGGCATTGTGGAAGG-3', backward primer 5' - GGATGCAGGGATGATGTTCT-3'. Final quantifications were calculated by the $2^{(-\triangle \Delta \mathrm{Ct})}$ method, and GAPDH was selected as a normalizing gene empirically. 


\section{Western blotting}

The Western blot assay was carried out as described previously [27]. Briefly, Proteins were resolved in SDSPAGE gels and immunoblotted with mouse pAbs against Cpn0423 (Fig. 1d), NOD2 and GAPDH (Fig. 4c) (Invitrogen) respectively. The primary antibody binding was probed with a horseradishperoxidase-conjugated goat anti-mouse IgG secondary antibody and was visualized with an enhanced chemiluminescence (ECL) kit (Sangon Biotech).

\section{Cpn0423 in vivo administration}

Male wild-type C57BL/6 mice (6-8 weeks old) were purchased from Model Animal Research Centre of Nanjing University. Mice were fed with bacteria-free diet and water. For Cpn0423 in vivo experiments, simple randomization method was used to assign animals to various experimental conditions. All mice used were gender and age matched. Firstly, Cpn0423 was pretreated with PTR according to the manufacture's instruction. WT mice were then administered with Cpn0423 pretreated with PTR $(50 \mathrm{mg} / \mathrm{kg})$, or phosphate-buffered saline (PBS), PTR alone, Cpn0423 (50 $\mathrm{mg} / \mathrm{kg}$ ) alone as controls through intranasal administration respectively. BALF and lung tissue were collected 15 days after Cpn0423 treatment as described [29]. Briefly, $1 \mathrm{ml}$ of PBS free of ionized calcium and magnesium was instilled four times via the tracheal cannula and was recollected by gentle manual aspiration. The fluids from each mouse were combined and immediately centrifuged at $700 \mathrm{~g}$ for $10 \mathrm{~min}\left(4{ }^{\circ} \mathrm{C}\right)$. The BALF supernatants were saved for cytokine measurements, and the cell pellets of BALF were suspended, manually counted and classified. The lung tissues were fixed with $4 \%$ paraformaldehyde fixative solution for histopathology examination, and the sections were stained with hematoxylin and eosin (H\&E). Inflammation scores in the lungs were performed in a blinded fashion using a reproducible scoring system, as previously described [30].
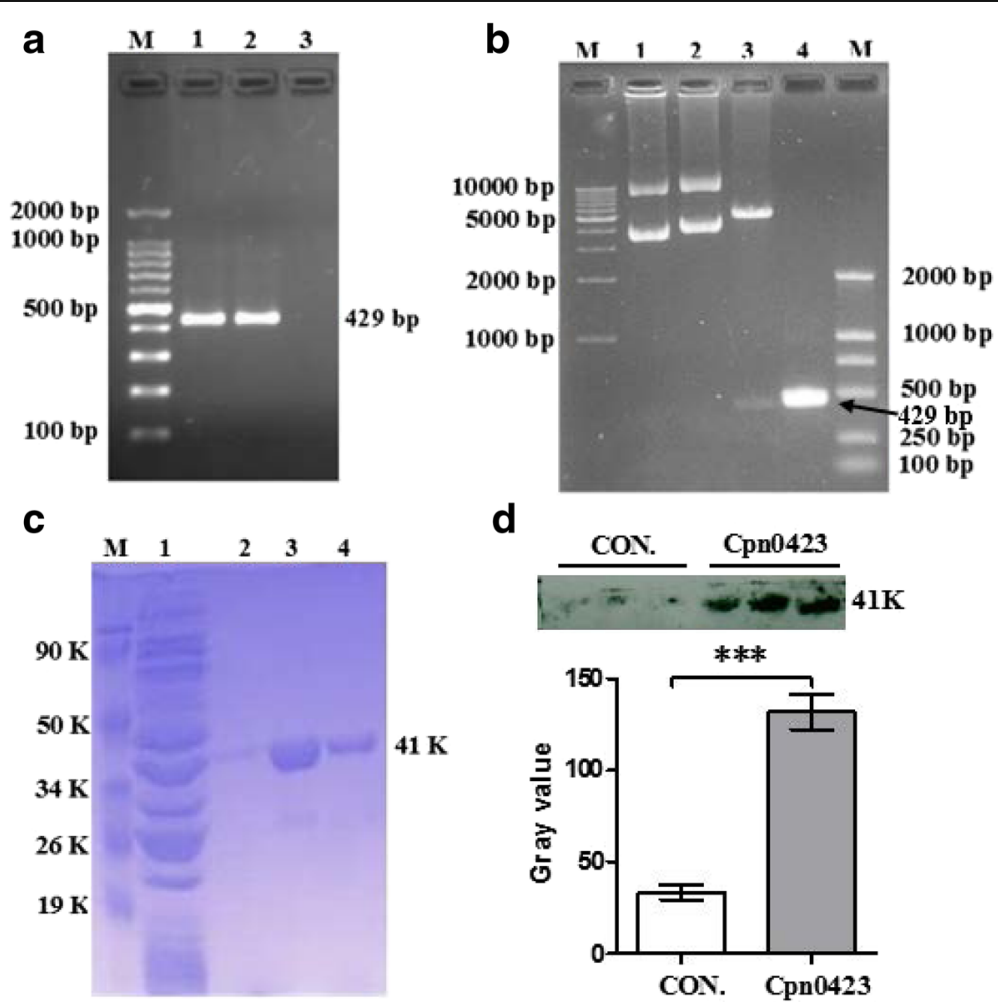

Fig. 1 Cloning, expression and purification of Cpn0423 of C. pneumoniae. a PCR products of Cpn0423 amplified from the C. pneumoniae strain AR-39. Lane M, DNA Marker; lanes 1 and 2, PCR amplified products of Cpn0423; lane 3, negative control. $\mathbf{b}$ Identification and restriction end nuclease analysis of the vector pGEX6p-2/Cpn0423. Lane M, DNA Marker; lane 1, pGEX6p-2 vector; lane 2, pGEX6p-2/Cpn0423 vector; lane 3, pGEX6p-2/Cpn0423 digested with Bam H I and Not I; lane 4, PCR amplified products of Cpn0423. c SDS-PAGE analysis of recombinant protein expressed in E. coli BL21 (DE3) cells. Lane M, protein marker; lane 1, supernatant fraction from cell lysate of bacteria transformed with pGEX6p-2/ Cpn0423 with IPTG (1 mM) induction for 4 h; lane 2, PBS Washings; lane 3: Protein Cpn0423 first eluent from glutathione sepharose; lane 4: Purified protein Cpn0423 from glutathione sepharose. $\mathbf{d}$ Immunoblotting of Cpn0423: eluent buffer (Con.) and purified Cpn0423 were immunoblotted for Cpn0423 


\section{Statistical analysis}

Statistical analysis was performed using Graphpad Prism 5 software (Graphpad, La Jolla, CA). Data were expressed as the mean $\pm \mathrm{SE}$, and statistical analysis of the data was performed using ANOVA and $t$ tests, Statistical differences were considered to be significant when $P<0.05$ for tests with one comparison.

\section{Results}

\section{Preparation of Cpn0423 fusion protein}

Cpn0423 gene was amplified by PCR using C. pneumoniae strain AR-39 genomic DNA. The specific primers amplified a 429 bp fragment (Fig. 1a). The digested PCR product (with Bam H I and Not I) was cloned into pGEX6p-2 vector and sequenced (Fig. 1b). The constructed vector, which expressed a soluble protein with the expected molecular mass of $41 \mathrm{kDa}$, was transformed into $E$. coli BL21cells. The recombinant protein was induced by IPTG and purified by using affinity chromatography (Fig. 1c). And the purified Cpn0423 protein reacted well with Cpn0423 polyclonal antibody (pAb) raised in this study (Fig. 1d). Endotoxin of purified recombinant protein used in this study was also removed by using commercial kits, and the final concentration of endotoxin was less than $60 \mathrm{pg} / \mathrm{ml}$ (Data not shown).

Cpn0423 is detected outside of C. pneumoniae inclusions Mice were immunized with the purified Cpn0423 fusion protein to raise polyclonal antibody (pAb), which were used to determine the localization of the endogenous Cpn0423 protein in C. pneumoniae-infected Hep-2 cells through immunofluorescence assay. As shown in Fig. 2a, the vast majority of Cpn0423 were detected in the cytosol of host cell infected with C. pneumoniae, but not in those of uninfected host cell, suggesting that Cpn0423 was mainly localized in the host cell cytosol, a pattern similar to CPAF protein (a chlamydial serine protease known to be secreted into the host cell cytosol), but distinct from those of chlamydial inclusion membrane protein [31]. We next confirmed the antibody labeling specificity by using an absorption procedure (Fig. 2b). The granular staining inside inclusion and the diffused staining in the host cell cytosol labeled by the anti-Cpn0423 antiserum was removed by absorption with GST-Cpn0423 but not GST-CPAF fusion proteins, demonstrating that anti-Cpn0423 antibodies specifically labeled the corresponding endogenous proteins without cross-reacting with CPAF. Resolving the precise identification and localization of Cpn0423 will require anti-Cpn0423 monoclonal (mAb) and techniques with higher resolution like confocal microscopy, which will be carried in the future. The above observations have demonstrated that Cpn0423 at least can be detected outside of C. pneumoniae inclusions.

\section{Cpn0423 induces cytokines production in BMDM}

To determine the effect of Cpn0423 on cytokines production, we treated BMDM with purified and
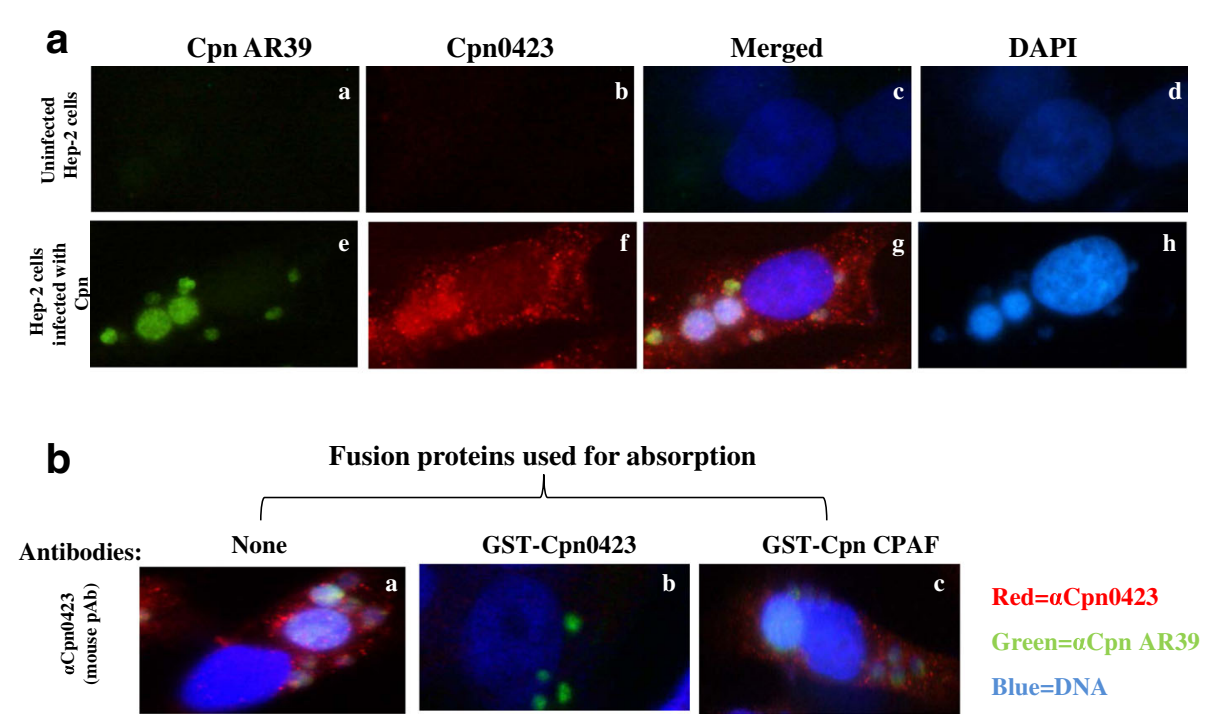

Fig. 2 Immunofluorescence detection of Cpn0423 in the C. pneumoniae-infected Hep-2 cells. Hep-2 cells infected with C. pneumoniae ( $A$, panels a-d) or uninfected Hep-2 cells ( $A$, panels $\mathbf{e}-\mathbf{h}$ ) were stained with mouse anti-Cpn0423 fusion protein antibodies visualized with a goat anti-mouse IgG-conjugated Cy3 (red). Cells were co-stained with a rabbit anti-Cpn AR39 visualized with a Cy2-conjugated goat anti-rabbit IgG (green) and a DNA Hoechst dye (b/ue). B, Cpn0423 pAb was pre-absorbed with Cpn0423and CPAF fusion proteins respectively, followed by immunostaining as described for panel A. The antibody pre-absorption was carried out as previously described [25]. Note that anti-Cpn0423 antibody labeling were removed by pre-absorption with the corresponding ( $B$, panel b) but not heterologous CPAF ( $B$, panel $\mathbf{c}$ ) fusion proteins 
endotoxins-removed Cpn0423. As shown in Fig. 3a, Cpn0423 induced an increase of MIP-2 protein production in a dose-dependent $(2.5-15 \mu \mathrm{g} / \mathrm{ml})$ manner at $16 \mathrm{~h}$, whilst it also induced a dosedependent $(2.5-15 \mu \mathrm{g} / \mathrm{ml})$ increase in IL-6 production at $16 \mathrm{~h}$ (Fig. 3b). Since endotoxins have strong biological effects even at very low concentrations in vivo and in vitro, we pretreated Cpn0423 with $\mathrm{PMB}$, a LPS neutralizer, to exclude the interference of any potential endotoxins contamination during Cpn0423 purification or treatment. Results showed in Fig. 3c confirmed that $\mathrm{PMB}$ effectively ruled out the possibility of potential LPS contamination without affecting MIP-2 response induced by Cpn0423. Furthermore, Cpn0423-induced cytokine production in BMDM was dependent on pretreatment with PTR, a liposome transfection reagent [32] that entered the cell via either direct fusion with the plasma membrane or by endocytosis and subsequent fusion with the endosome, releasing the captured protein into the cytoplasm (Fig. 3d). In all following experiments, we used $10 \mu \mathrm{g} / \mathrm{ml}$ of Cpn0423 complexed with PTR.

\section{Cpn0423-induced cytokine response is attenuated by NOD2-siRNA}

The cytosolic pattern recognition receptor NOD2 can be activated to generate a proinflammatory immune responses, and the early immune response involves the influx of macrophages [6]. We hypothesized that NOD2 signaling was responsible for the Cpn0423-induced cytokine production. To test the hypothesis, we synthesized the specific NOD2-siRNA and NOD2-Con-siRNA oligonucleotides. We performed quantitative PCR and western blot to determine the mRNA and protein expression levels of NOD2 receptor in BMDM. As shown in Fig. 4a, $\mathrm{b}$ and $\mathrm{c}$, compared to the NOD2-Con-siRNA oligonucleotide sequence, NOD2-siRNA pretreatment resulted in significant, but incomplete, reduction of NOD2. NOD2-siRNA also reduced NOD2 receptor mRNA level induced by MDP and attenuated NOD2 protein expression induced by Cpn0423, respectively. However, the mRNA level of TLR2 receptor expression induced by P3C, a TLR2 ligand, was unaffected. Of note, Cpn0423 induced NOD2, but not TLR2, the expression in BMDM, and the specificity of NOD2-siRNA was
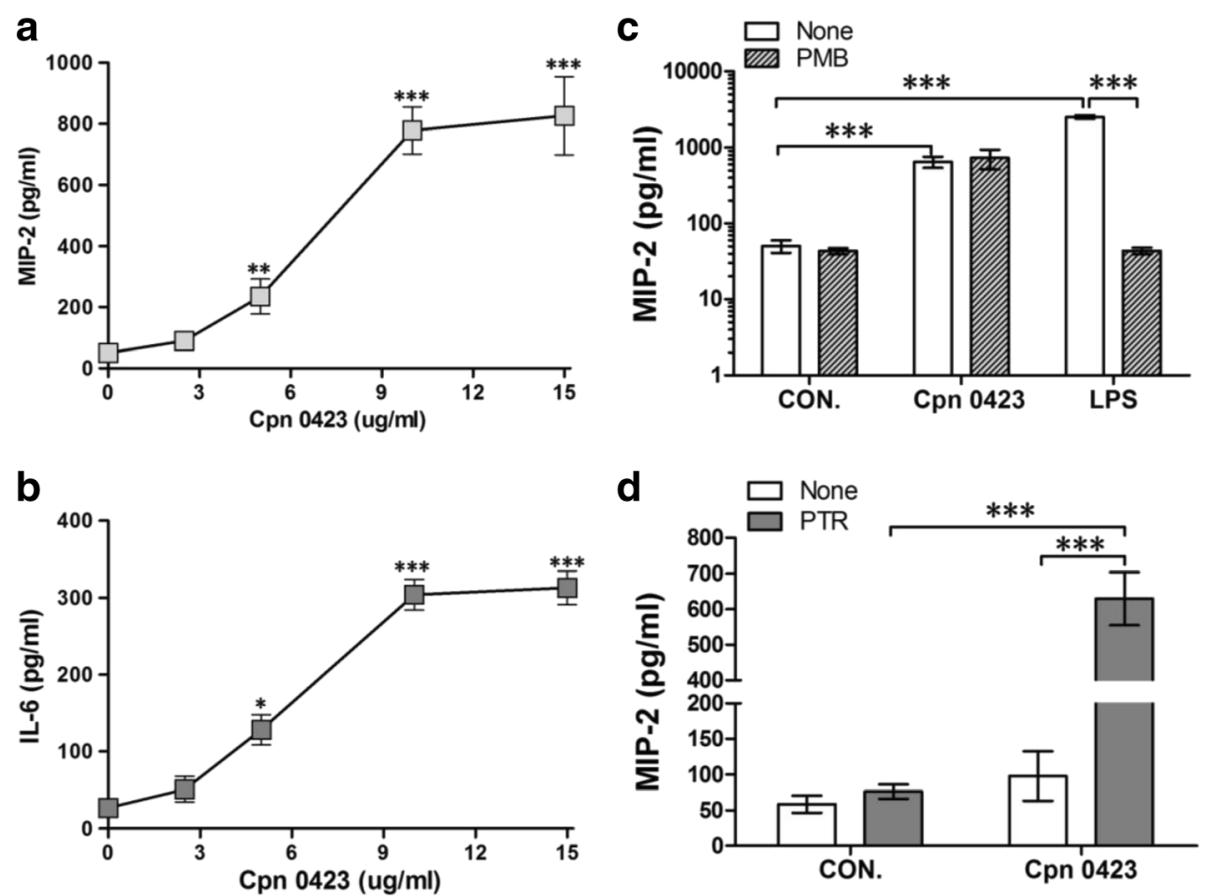

Fig. 3 Cpn0423 fusion protein induces cytokine production. Bone marrow-derived macrophages (BMDMs, A-C) were treated with Cpn0423 in the presence of PTR. Cells plated in 96-wells were treated for 18-24 $\mathrm{h}$ and the media were harvested for cytokines protein measurement. a Dose course of Cpn0423-induced MIP-2 production in BMDMs. $\mathbf{b}$ Dose course of Cpn0423-induced IL-6 production in BMDMs. $\mathbf{c}$ Effect of polymyxin B sulfate (PMB, a LPS neutralizer) on Cpn0423 MIP-2 production. Cpn0423 (10 $\mu \mathrm{g} / \mathrm{ml})$ or LPS (10 ng/ml) was incubated with $50 \mu \mathrm{g} / \mathrm{ml}$ PMB for 30 min at $4{ }^{\circ} \mathrm{C}$ before treatment. $\mathbf{d}$ Effect of PTR on Cpn0423-induced MIP-2 production. BMDMs were treated with $10 \mu \mathrm{g} / \mathrm{ml} \mathrm{Cpn0423}$ pre-treated with or without PTR (None group). Each error bar represented Mean \pm SD. Two-tailed unpaired student $t$ test was used in Fig. 3A-D, ${ }^{*} P<0.05,{ }^{* *} P<0.01,{ }^{* * *} P<0.001 . n=3$ in each group. PMB: polymyxin B sulfate; PTR: Protein Transfection Reagent 


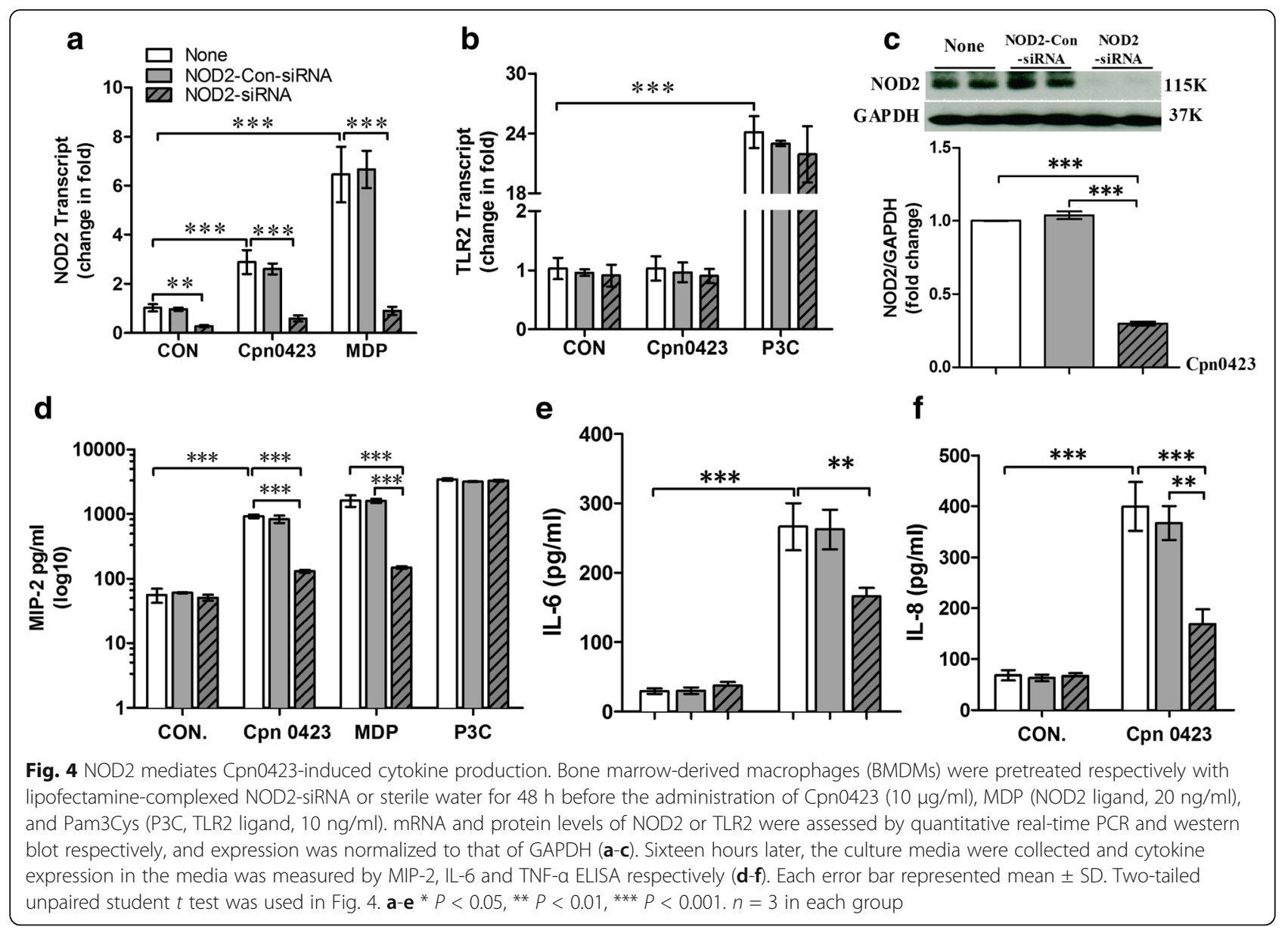

confirmed by its selective inhibitory effect on MDPinduced mRNA expression of NOD2.

To further test the impact of NOD2 signaling on Cpn0423-induced cytokine responses, we treated BMDM with Cpn0423, NOD2 and TLR2 ligand. As anticipated, Cpn0423 and NOD2 induced robust MIP-2 protein production in BMDMs (Fig. 4d). However, MIP2 protein production was lessened significantly but incompletely by about $89.7 \%$ and $85.6 \%$, respectively, after NOD2-siRNA treatment, while P3C-induced MIP2 protein production remained unaffected. We also tested other cytokines productions in responses to Cpn0423. Similarly, Cpn0423-induced IL-6 and TNF- $\alpha$ productions were significantly decreased in BMDMs pretreated with NOD2-siRNA, but remained the same after NOD2-Con-siRNA pretreatment (Fig. 4e and f).

Intranasal Cpn0423 induces pulmonary inflammation in vivo To evaluate the ability of $\mathrm{Cpn} 0423$ to induce pulmonary inflammation in vivo, mice were treated with Cpn0423 pretreated with PTR, Cpn0423 alone, PBS and PTR alone via intranasal administration respectively. Histopathology analysis of lung sections 15 days after administration showed there was no histological evidence of inflammation in PBS and PTR control group mice, but detectable inflammation could be observed in Cpn0423 and Cpn0423 pretreated with PTR group mice (Fig. 5a). As shown in Fig. 5b, Cpn0423 significantly increased inflammatory scores significantly compared to PBS and PTR control group $(P<0.01)$, which was markedly enhanced by Cpn0423 pre-treatment with PTR (Fig. 5b), suggesting an essential role of PTR on Cpn0423-induced inflammatory response.

To investigate the effect of Cpn0423 on leukocyte recruitment, we calculated the total cell number of BALF in mice as presented in Fig. 5c. Evaluation showed no significant differences in total cell number in PBS $\left(0.43 \pm 0.13 \times 10^{5}\right)$ and PTR group $\left(0.50 \pm 0.43 \times 10^{5}\right)$ $(P>0.05)$, while total cell increase in BALF was observed in Cpn0423 group $\left(0.76 \pm 0.35 \times 10^{5}\right)$, and the cell number increase to $2.32 \pm 0.42 \times 10^{5}$ after PTR pre-treatment $(P<0.01)$. BALF cell classification revealed a decrease in macrophages $(88 \pm 4.02 \%$ vs. $56.18 \% \pm 4.04 \%, P<0.001)$ and an increase in polymorphonuclear leukocyte (PMN) $(9.18 \pm 2.79 \%$ vs. $27.56 \pm 12.61 \%, P<0.01)$ in the Cpn0423 administered 

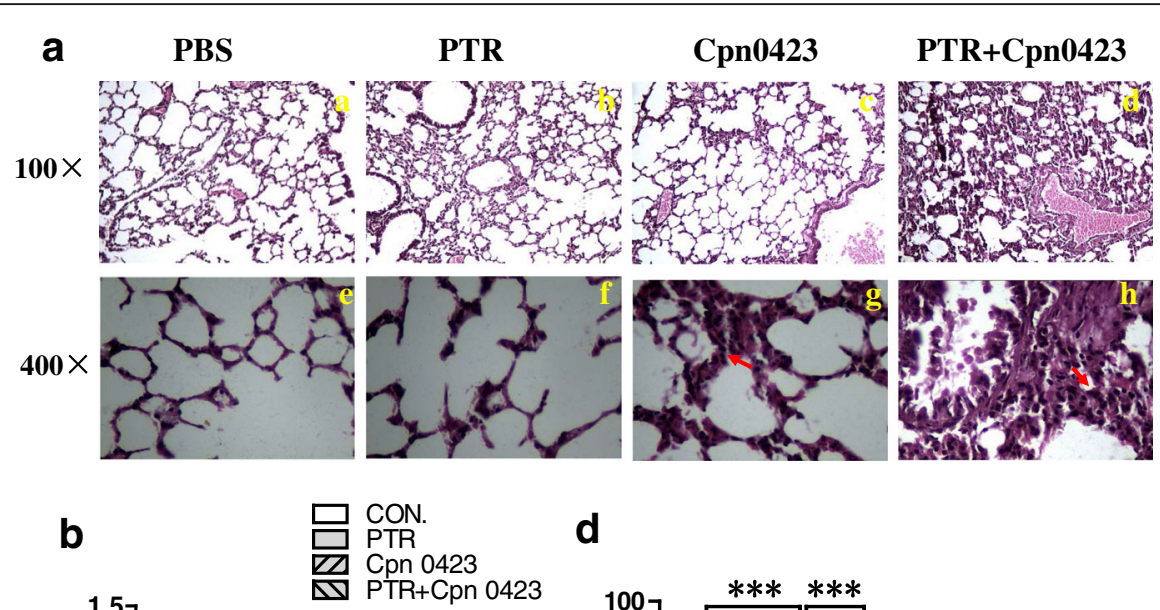

d
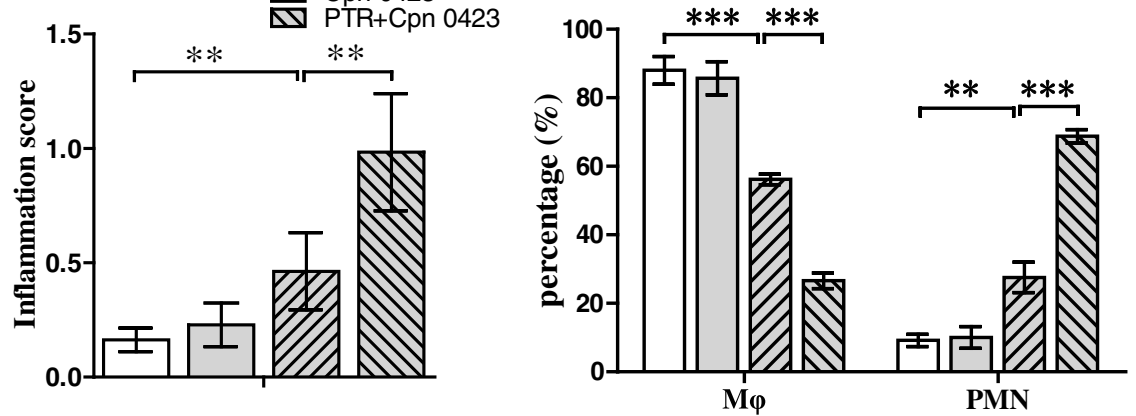

C

e
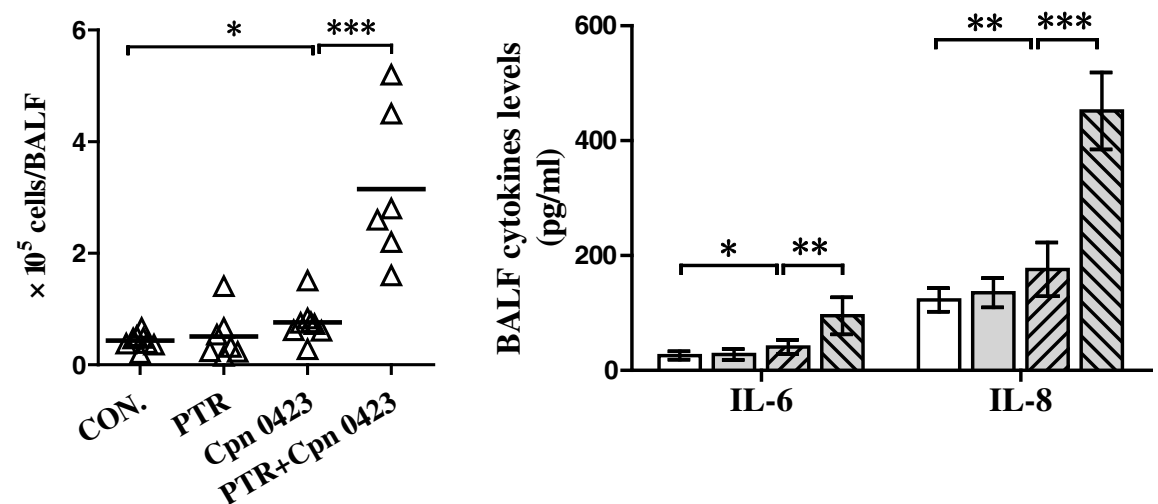

Fig. 5 Effects of Cpn0423 on pulmonary inflammation in vivo. A, Histopathological analysis of lung tissues from mice of all the experimental groups were performed at days 15 after intranasal administration (haematoxylin and eosin staining, panels a-d magnification 100x; panels e-h magnification 400x). Mice were treated with PBS (a \& e), PTR (b \& f), Cpn0423 (50 mg/kg) (c \& g), and PTR + Cpn0423 (50 mg/kg) (d \& h). $B$, Results of histological scoring for pulmonary inflammatory changes of lung sections from different groups of mice. A score of 0-3 was adjudged to the tissue sections of the mice as described in Materials and methods. C, The total BALF cells in each group. D, The percentage of macrophages $(M \varphi)$ and polymorphonuclear leukocyte (PMN) among total BALF cells. E, Levels of the cytokines IL-6 and TNF- $a$ in BALF were measured using enzyme-linked immunosorbent assay. Each error bar represented mean \pm SD. Two-tailed unpaired student $t$ test was used in Fig. 5. $E-H,{ }^{* *} P<0.01,{ }^{* * *} P<0.001 . n=8$ mice in PBS Group, $n=7$ mice in PTR-control Group, $n=8$ mice in Cpn0423 group, $n=6$ mice in Cpn0423 + PTR group. PTR: Protein Transfection Reagent. Red arrowhead indicates inflammatory cells infiltration

mice compared to the control mice with PBS treatment. Similarly, PTR pre-treatment significantly improved the role of Cpn0423 on macrophages $(26.58 \% \pm 5.62 \%, P<0.001)$ and PMN $(68.75 \% \pm 4.71 \%$, $P<0.001$ ) cell classification (Fig. $5 \mathrm{~d}$ ), suggesting that Cpn0423 induced inflammatory cells recruitment and affected the ratio of macrophages and PMN. To gain insight into the effect of Cpn0423 on inflammation, we detected the changes of IL- 6 and TNF- $\alpha$ in the BALF supernatant. As expected, intranasal administration of Cpn0423 or Cpn0423 pre-treated with PTR both resulted in a significant increase in IL-6 $(P<0.05$ $\& P<0.01)$ and TNF- $\alpha(P<0.01 \& P<0.001)$ levels in the BALF (Fig. 5e). These data indicated that Cpn0423 
induced pulmonary inflammation in vivo, consistent with our in vitro observations.

\section{Discussion}

Chronic C. pneumoniae infections have been associated with the induction or the acceleration of various inflammatory diseases [33]. Although a few studies have demonstrated that C. pneumoniae heat shock protein 60 (HSP60) activates NF-kB in a MyD88-independent TLR4 manner [34], the cellular and molecular mechanisms by which $C$. pneumoniae participates in the inflammatory responses remain to be elucidated. Hence it is important to identify the exact components of C. pneumoniae and the pattern recognition receptors which initiate or are involved in the inflammatory responses. In the current study, we made three main findings. First, we found that Cpn0423 was detected in the cytosol of C. pneumoniaeinfected cells, and we detected robust cytokine responses in BMDMs when they were treated with Cpn0423. Second, NOD2 signaling was involved in the Cpn0423induced MIP-2 expression. Third, Cpn0423 intranasal administration in vivo induced pulmonary inflammation and caused pneumonia.

C. pneumoniae are obligate intracellular parasites that only live inside the host cells to ensure their successful survival, which has been shown to infect and productively replicate within a number of cell types [5], including macrophages. Therefore, the components of $C$. pneumoniae might fulfill its function mostly inside the host cells. Considering that, we transfected Cpn0423 into BMDMs using lipid-based PTR, and our data demonstrated that the cytokine-producing effect of Cpn0423 was markedly enhanced and dependent on the presence of PTR.

Endotoxins liberated by gram-negative bacteria are frequent contaminations of protein solutions derived from bioprocesses and showed strong biological effects at very low concentrations [35]. Therefore, their removal is essential for recombinant proteins administration. Herein, we conducted the removal of LPS from Cpn0423 by Endotoxin Removal Resin. Our data showed that PMB pretreatment totally diminished LPS-induced MIP-2; in contrast, it did not affect Cpn0423-induced MIP-2 response, suggesting efficient endotoxin removal from Cpn0423 in our study.

Innate immunity is an evolutionarily ancient part, which lies behind most inflammatory responses. Innate immune recognition relies on a limited number of germline-encoded receptors, including cell surface receptors, TLR2, TLR4, and TLR11, and intracellular pattern recognition receptors, e.g. NOD2, TLR3, and TLR9 [13, 15]. Recent studies strongly support an important role of C. pneumoniae HSPs, LPS and MOMP played in driving innate immune responses [16, 36], while TLR2 and TLR4 are both important to orchestrate cytokine and chemokine responses and host defense against C. pneumoniae infection in vivo. TLR3 and TLR9 are located primarily on the membranes of intracellular compartments and mainly responsible for the recognition of microbial nucleic acids [13]. NOD2 is a kind of cytosolic proteins that respond to intracellular fragments of bacterial peptidoglycan, which is also crucial for innate immune responses to certain bacterial infections $[13,20]$. C. pneumoniae can gain access to the intracellular compartments, whereas the link between $C$. pneumoniae and NOD2 has been barely studied. In this study, Cpn0423 was detected in the cytosol of C. pneumoniae-infected cells, and the detection of the endogenous Cpn0423 was specific. The anti-Cpn0423 antibody labeling observed under the fluorescence microscope was only removed by absorption with Cpn0423 but not CPAF fusion protein. Moreover, we characterized NOD2 expression in BMDMs as well as the specificity of NOD2-siRNA, and found that NOD2-siRNA pretreatment dramatically attenuated Cpn0423-induced cytokine responses in BMDMs, suggesting that NOD2 was involved in Cpn0423-induced cellular inflammation. Although our results suggest a role for NOD2 in Cpn0423-induced inflammatory response, the exact downstream signaling cascade remains to be elucidated. One candidate molecule is the receptor interacting protein kinase 2 [37], which has been suggested to link NOD2 and efficient immune responses during C. pneumoniae infection.

C. pneumoniae infection might contribute to chronic inflammatory events associated with pneumonia [38], whereas, the exact components of C. pneumoniae which activate immune system in vivo have been barely studied. In the present study, we have presented convincing evidence that $C$. pneumoniae protein Cpn0423 could cause pneumonia and induce a strong immune response. After Cpn0423 intranasal administration, we observed increased infiltrating inflammatory cells in the lung histology, recruitment of a large number of immune cells with a higher percentage of neutrophils and lower percentage of macrophages, and higher cytokines levels in the BALF. Macrophages, as resident cells of almost every tissue in the body, may be stimulated first by Cpn0423, and secreted chemokines such as MIP-2 and IL-8 to trigger neutrophils accumulation in a cascade amplification way $[39,40]$. This, may explain the proportion changes of neutrophils and macrophages. Interestingly, unlike in vitro, inflammatory response was also found in mice administered with Cpn0423 alone, the possible reason maybe some compounds in the body could help Cpn0423 enter into the host cells to fulfil its function. 
We detected marked cytokine responses both in vitro and in vivo upon treatment with Cpn0423. Whether the dose of Cpn0423 used in this study corresponded to the one generated during a natural infection remains unknown. However, C. pneumonia infection has a gradual onset, and the organism often reside intracellularly and secrete various proteins with different quantities for indefinite periods, potentially promoting chronic inflammation [4, 41]. To the best of our knowledge, it is difficult to evaluate how much $C$. pneumonia protein can be secreted from infected host cells or what C. pneumonia protein amount are presented in infected cells. Thus, the concentration of Cpn0423 used in this paper was based on our pilot studies and previous reported $[16,34]$. Here we showed for the first time the discovery of recombinant protein Cpn0423 as an activator and its effects on pneumonia. We will continue to clarify the mechanism of entry and presentation of Cpn0423 to NOD2, the precise NOD2 downstream molecular signaling in Cpn0423-induced inflammation, and the role of endogenous Cpn0423 during C. pneumoniae infection.

\section{Conclusions}

In the present paper, we demonstrated that C. pneumoniae protein could act as signals for intracellular pattern recognition receptors activation. Specially, we found that Cpn0423 was an activator of NOD2 and provoked profound inflammatory responses in BMDMs. We also identified the pivotal role of Cpn0423 in mediating pulmonary inflammation in vivo. We speculated that Cpn0423 exists under during $C$. pneumoniae infection conditons and stimulated NOD2, thereby accelerating host immune responses against $C$. pneumoniae. These observations suggest that $\mathrm{Cpn} 0423$ is a potent and specific innate immune activator that may initiate a local inflammation by inducing cytokine release. Further characterization of the inflammatory effect of C. pneumoniae protein will speed up our understanding of the pathogenic mechanism of $C$. pneumoniae.

\section{Abbreviations}

BALF: Bronchoalveolar lavage fluid; BMDM: bone marrow-derived macrophages; Cpn: Chlamydia pneumoniae; ll: interleukin; MDP: muramyl dipeptide; MIP-2: macrophage inflammatory protein-2; NOD2: Nucleotide-binding oligomerization domain-containing protein 2; NOD2-Con-siRNA: small interfering RNA negative control for NOD2; NOD2-siRNA: small interfering RNA against NOD2; P3C: Pam ${ }_{3}$ Cys-Ser-(Lys) 4 ; TLR: Toll-like receptors

\section{Acknowledgements}

We are grateful to Dr. Guang-Ming Zhong for providing C. pneumoniae strain AR39, Dr. Chun-xue Lu and Mr. Chen-xu Guo for assisting with manuscript revision.

\section{Funding}

This work was supported by the Science Foundation of First People's Hospital of ChenZhou (No. N2013-006), Hunan Provincial Natural Science Foundation of China (2017JJ3286), the Foundation of Hunan Provincial Key
Laboratory for Special Pathogens Prevention and Control Foundation (No. 2014-5) and the Foundation of Chenzhou municipal Science and Technology Bureau (CZ2014029).

\section{Availability of data and materials}

The dataset supporting the conclusions of this article was included within the article.

\section{Authors' contributions}

HC conceived and designed the study, performed the experiments in Figs. 1, 2, 3, 4 and 5, analyzed the data, and drafted the manuscript. GD, AZ and HY performed the experiments shown in Figs. 1 and 2. RL, OR, XY and JX performed the experiments and analyzed the data shown in Figs. 3, 4 and 5. YW is the principal investigator and responsible for all aspects of the project. All authors reviewed the results and approved the final version of the manuscript.

Ethics approval and consent to participate

The animal protocols were approved by the Ethics Committee of University of South China (ethics approval reference number: NH20140329).

\section{Consent for publication}

Not applicable.

\section{Competing interests}

The authors declare that they have no conflicts of interest with the contents of this article.

\section{Publisher's Note}

Springer Nature remains neutral with regard to jurisdictional claims in published maps and institutional affiliations.

\section{Author details}

'Department of Clinical Laboratory, ChenZhou NO.1 People's Hospital, ChenZhou 423000, China. ${ }^{2}$ Institute of Translational Medicine, University of South China, ChenZhou 423000, China. ${ }^{3}$ Institute of Pathogenic Biology, Medical College, University of South China, Hengyang 421001, China.

Received: 18 October 2016 Accepted: 29 June 2017

Published online: 11 July 2017

\section{References}

1. Holter JC, Muller F, Bjorang O, Samdal HH, Marthinsen JB, Jenum PA, Ueland T, Froland SS, Aukrust P, Husebye E, et al. Etiology of community-acquired pneumonia and diagnostic yields of microbiological methods: a 3-year prospective study in Norway. BMC Infect Dis. 2015;15:64.

2. Hahn DL, Dodge RW, Golubjatnikov R. Association of Chlamydia pneumoniae (strain TWAR) infection with wheezing, asthmatic bronchitis, and adult-onset asthma. JAMA. 1991:266(2):225-30.

3. Al-Aydie SN, Obeidat NM, Al-Younes HM. Role of Chlamydia pneumoniae in community-acquired pneumonia in hospitalized Jordanian adults. J Infect Dev Countries. 2016;10(3):227-36.

4. Jupelli M, Shimada K, Chiba N, Slepenkin A, Alsabeh R, Jones HD, Peterson E, Chen S, Arditi M, Crother TR. Chlamydia pneumoniae infection in mice induces chronic lung inflammation, iBALT formation, and fibrosis. PLoS One. 2013:8(10):e77447.

5. Mukhopadhyay S, Good D, Miller RD, Graham JE, Mathews SA, Timms P, Summersgill JT. Identification of Chlamydia pneumoniae proteins in the transition from reticulate to elementary body formation. Mol Cell Proteomics. 2006:5(12):2311-8.

6. Shimada K, Chen S, Dempsey PW, Sorrentino R, Alsabeh R, Slepenkin AV, Peterson E, Doherty TM, Underhill D, Crother TR, et al. The NOD/RIP2 pathway is essential for host defenses against Chlamydophila pneumoniae lung infection. PLoS Pathog. 2009;5(4):e1000379.

7. He X, Mekasha S, Mavrogiorgos N, Fitzgerald KA, Lien E, Ingalls RR. Inflammation and fibrosis during Chlamydia pneumoniae infection is regulated by IL-1 and the NLRP3/ASC inflammasome. J Immunol. 2010; 184(10):5743-54.

8. Kuo CC, Jackson LA, Campbell LA, Grayston JT. Chlamydia pneumoniae (TWAR). Clin Microbiol Rev. 1995;8(4):451-61.

9. Grayston JT. Infections caused by Chlamydia pneumoniae strain TWAR. Clin Infect Dis. 1992;15(5):757-61. 
10. Mehta SJ, Miller RD, Ramirez JA, Summersgill JT. Inhibition of Chlamydia pneumoniae replication in HEp-2 cells by interferon-gamma: role of tryptophan catabolism. J Infect Dis. 1998;177(5):1326-31.

11. Fan $\mathrm{P}$, Dong $F$, Huang $Y$, Zhong G. Chlamydia pneumoniae secretion of a protease-like activity factor for degrading host cell transcription factors required for [correction of factors is required for] major histocompatibility complex antigen expression. Infect Immun. 2002;70(1):345-9.

12. Kalman S, Mitchell W, Marathe R, Lammel C, Fan J, Hyman RW, Olinger L, Grimwood J, Davis RW, Stephens RS. Comparative genomes of Chlamydia pneumoniae and C. Trachomatis. Nat Genet. 1999;21(4):385-9.

13. Brubaker SW, Bonham KS, Zanoni I, Kagan JC. Innate immune pattern recognition: a cell biological perspective. Annu Rev Immunol. 2015;33:257-90

14. Hoffmann JA, Kafatos FC, Janeway CA, Ezekowitz RA. Phylogenetic perspectives in innate immunity. Science. 1999;284(5418):1313-8.

15. Palm NW, Medzhitov R. Pattern recognition receptors and control of adaptive immunity. Immunol Rev. 2009;227(1):221-33.

16. Zhou Z, Wu Y, Chen L, Liu L, Chen H, Li Z, Chen C. Heat shock protein 10 of Chlamydophila pneumoniae induces proinflammatory cytokines through toll-like receptor (TLR) 2 and TLR4 in human monocytes THP-1. In Vitro Cell Dev Biol Animal. 2011;47(8):541-9

17. Shimada K, Crother TR, Arditi M. Innate immune responses to Chlamydia pneumoniae infection: role of TLRs, NLRs, and the inflammasome. Microbes Infect. 2012;14(14):1301-7.

18. Rothfuchs AG, Trumstedt C, Wigzell H, Rottenberg ME. Intracellular bacterial infection-induced IFN-gamma is critically but not solely dependent on toll-like receptor 4-myeloid differentiation factor 88-IFN-alpha beta-STAT1 signaling. J Immunol. 2004;172(10):6345-53.

19. Philpott DJ, Sorbara MT, Robertson SJ, Croitoru K, Girardin SE. NOD proteins: regulators of inflammation in health and disease. Nat Rev Immunol. 2014; 14(1):9-23.

20. Hsu LC, Ali SR, McGillivray S, Tseng PH, Mariathasan S, Humke EW, Eckmann $L$, Powell JJ, Nizet V, Dixit VM, et al. A NOD2-NALP1 complex mediates caspase-1-dependent IL-1beta secretion in response to bacillus anthracis infection and muramyl dipeptide. Proc Natl Acad Sci U S A. 2008;105(22): 7803-8

21. Ferwerda $G$, Kramer $M$, de Jong D, Piccini A, Joosten LA, Devesaginer I, Girardin SE, Adema GJ, van der Meer JW, Kullberg BJ, et al. Engagement of NOD2 has a dual effect on prolL-1 beta mRNA transcription and secretion of bioactive IL-1beta. Eur J Immunol. 2008;38(1):184-91.

22. Luo J, Jia T, Zhong Y, Chen D, Flores R, Zhong G. Localization of the hypothetical protein Cpn0585 in the inclusion membrane of Chlamydia pneumoniae-infected cells. Microb Pathog. 2007;42(2-3):111-6.

23. Hongliang C, Zhou Z, Zhan H, Yanhua Z, Zhongyu L, Yingbiao L, Guozhi D, Yimou W. Serodiagnosis of Chlamydia pneumoniae infection using three inclusion membrane proteins. J Clin Lab Anal. 2010;24(1):55-61.

24. Sharma J, Zhong Y, Dong F, Piper JM, Wang G, Zhong G. Profiling of human antibody responses to Chlamydia trachomatis urogenital tract infection using microplates arrayed with 156 chlamydial fusion proteins. Infect Immun. 2006;74(3):1490-9.

25. Lei L, Qi M, Budrys N, Schenken R, Zhong G. Localization of Chlamydia trachomatis hypothetical protein CT311 in host cell cytoplasm. Microb Pathog. 2011:51(3):101-9.

26. Zheng J, Ding T, Chen Z, Fang H, Li H, Lu H, Wu Y. Preparation and evaluation of monoclonal antibodies against chlamydial protease-like activity factor to detect Chlamydia pneumoniae antigen in early pediatric pneumonia. Eur J Clin Microbiol Infect Dis. 2015;34(7):1319-26.

27. Feng Y, Chen H, Cai J, Zou L, Yan D, Xu G, Li D, Chao W. Cardiac RNA induces inflammatory responses in Cardiomyocytes and immune cells via toll-like receptor 7 signaling. J Biol Chem. 2015;290(44):26688-98.

28. Cavaillon JM. Polymyxin B for endotoxin removal in sepsis. Lancet Infect Dis. 2011;11(6):426-7.

29. Tournoy KG, Kips JC, Schou C, Pauwels RA. Airway eosinophilia is not a requirement for allergen-induced airway hyperresponsiveness. Clin Experimental Allergy. 2000;30(1):79-85.

30. Naiki Y, Michelsen KS, Schroder NW, Alsabeh R, Slepenkin A, Zhang W, Chen $\mathrm{S}$, Wei $\mathrm{B}$, Bulut $\mathrm{Y}$, Wong $\mathrm{MH}$, et al. MyD88 is pivotal for the early inflammatory response and subsequent bacterial clearance and survival in a mouse model of Chlamydia pneumoniae pneumonia. J Biol Chem. 2005: 280(32):29242-9.
31. Zhong G, Fan P, Ji H, Dong F, Huang Y. Identification of a chlamydial protease-like activity factor responsible for the degradation of host transcription factors. J Exp Med. 2001;193(8):935-42.

32. Zelphati O, Szoka FC Jr. Mechanism of oligonucleotide release from cationic liposomes. Proc Natl Acad Sci U S A. 1996;93(21):11493-8.

33. Grayston JT, Campbell LA, Kuo CC, Mordhorst CH, Saikku P, Thom DH, Wang SP. A new respiratory tract pathogen: Chlamydia pneumoniae strain TWAR. $J$ Infect Dis. 1990;161(4):618-25.

34. Bulut $Y$, Shimada $K$, Wong MH, Chen S, Gray P, Alsabeh R, Doherty TM, Crother TR, Arditi M. Chlamydial heat shock protein 60 induces acute pulmonary inflammation in mice via the toll-like receptor 4- and MyD88dependent pathway. Infect Immun. 2009;77(7):2683-90.

35. Esteban E, Ferrer R, Alsina L, Artigas A. Immunomodulation in sepsis: the role of endotoxin removal by polymyxin B-immobilized cartridge. Mediat Inflamm. 2013. doi:10.1155/2013/507539.

36. Schumacher A, Seljeflot I, Lerkerod AB, Sommervoll L, Otterstad JE, Arnesen H. Chlamydia LPS and MOMP seropositivity are associated with different cytokine profiles in patients with coronary heart disease. Eur J Clin Investig. 2005:35(7):431-7.

37. Wagner RN, Proell M, Kufer TA, Schwarzenbacher R. Evaluation of nod-like receptor (NLR) effector domain interactions. PLoS One. 2009;4(4):e4931.

38. Huston WM, Barker CJ, Chacko A, Timms P. Evolution to a chronic disease niche correlates with increased sensitivity to tryptophan availability for the obligate intracellular bacterium Chlamydia pneumoniae. J Bacteriol. 2014; 196(11):1915-24.

39. Wynn TA, Chawla A, Pollard JW. Macrophage biology in development, homeostasis and disease. Nature. 2013;496(7446):445-55.

40. Weckmann M, Becker T, Nissen G, Pech M, Kopp MV. SiMA: a simplified migration assay for analyzing neutrophil migration. Cytometry A. 2017. doi:10.1002/cyto.a.23114

41. Hogan RJ, Mathews SA, Mukhopadhyay S, Summersgill JT, Timms P. Chlamydial persistence: beyond the biphasic paradigm. Infect Immun. 2004; 72(4):1843-55.

\section{Submit your next manuscript to BioMed Central and we will help you at every step:}

- We accept pre-submission inquiries

- Our selector tool helps you to find the most relevant journal

- We provide round the clock customer support

- Convenient online submission

- Thorough peer review

- Inclusion in PubMed and all major indexing services

- Maximum visibility for your research

Submit your manuscript at www.biomedcentral.com/submit 